\title{
Presentation mode differences in the auditory discrimination of retarded children'
}

\author{
JEROME SMITH, University of Connecti- \\ cut, Storrs, Conn. 06268
}

The effects of presentation mode (simultaneous or successive and stimulus-response spatial contiguity or noncontiguity/ upon the auditory discrimination of retarded children are reported. $S-R$ spatial contiguity appears to produce better performance, but simultaneous-successive differences are not found. The interactions among presentation modes found in previous visual discrimination studies are not present in the auditory situation.

In a comparison of auditory and visual discrimination learning in retarded children, Smith, Anderson, Cunningham, \& Sjoberg (1967) found that although the shapes of the acquisition functions in both sense modes were similar, there appeared to be differential effects of presentation mode. In the visual situation simultaneous presentation of both discriminanda, coupled with spatial contiguity of the stimulus and response, produced superior performance to a condition in which the discriminanda were presented successively and the stimulus and response were separated spatially. Such a presentation mode difference in visual discrimination has precedent with both normal (Lipsitt, 1961) and retarded (Shepp, 1960) children. The auditory discrimination in the experiment of Smith et al involved successive presentation and spatial separation of the stimulus and response. It was therefore similar to the successive visual problem. The ease of solution of the auditory problem, howver, was closer to the simultaneous visual problem than to the successive visual problem. It was suggested that perhaps presentation mode effects in the visual and auditory senses were different. The training history of the child was offered as a possible rationale for such differences. "While simultaneous visual discriminations are fairly common for the child, auditory discriminations are probably successive for the most part ...."

The present experiment was designed to determine the effects of presentation mode upon auditory discrimination. Lipsitt (1961) has shown that for normal children simultaneous visual discriminations are easier than successive ones when stimulus and responses are spatially contiguous, but that this effect is eliminated or possibly reversed when the locus of response is removed from the stimulus. That these effects appear to hold for retarded children may be inferred from the experiments of Shepp (1960) and Smith et al (1967). The differential training history hypothesis offered earlier would suggest that equivalent effects would not be found in auditory discriminations. If successive discriminations were the most common auditory experience, one would not necessarily expect simultaneous superiority under either stimulus-response spatial contiguity or separation. It is possible, moreoever, that successive discrimination would prove easier under both conditions. The present experiment utilizes a factorial design to test the effects upon retardate auditory discrimination of two independent variables: successive vs simultaneous presentation, and S-R spatial contiguity vs spatial separation of S-R.

The simultaneous-successive distinction in the visual situation is simple to implement. In a simultaneous discrimination the child is given both discriminanda and a response is made in the "correct" one after both have been inspected. In the successive situation only one object is presented and the child makes a "correct" response (go left). He makes a different response (go right) for the other object presented on a different trial. The crucial difference is that in the simultaneous situation both objects appear together before the response is made, while in the successive situation only one object precedes the response. In auditory discrimination the problem is different to some extent. Presentation of two sounds simultaneously can produce masking and other interactive effects which may produce confusion, particularly in the retarded child. The simultaneous condition in the present experiment preserves its similarity to the visual situation by having both sounds presented before the response, but eliminates interactive effects by having one sound immediately followed by a second with no overlap. The successive situation is, of course, directly analogous to the visual.

\section{SUBJECTS}

Forty-four retarded males and females at the Mansfield State Training School, Mansfield, Connecticut, served as Ss. All Ss had normal hearing and exhibited no major visual or motor impairment. All Ss had prior experience in simultaneous visual discrimination experiments, but were naive with respect to auditory discrimination studies. APPARATUS

A modified Wisconsin General Test Apparatus (Zeaman \& House, 1963) was used for presentation of stimuli. Reinforcement wells were covered by two identical small speakers from which emanated the sounds for the S-R spatial contiguity conditions. When the stimuli and response were spatially separated, sounds were delivered through stereophonic earphones. The speakers were still present but served as covers for the reinforcement wells.

Sounds were produced by a Sony tape deck and amplifier and fed to either the speaker or earphones. The sound tape consisted of sequences of "junk" sounds each lasting for $60 \mathrm{sec}$. The "junk" sounds differed in several stimulus dimensions and consisted of a pool of six sounds: the bark of a dog, a boat whistle, the gallop of horse hoofs, a siren, the clash of a cymbal, and a buzzer. For the simultaneous condition one stimulus was presented on one stereophonic channel, followed immediately by the other stimulus on the other stereo channel. For successive presentation the same stimulus was recorded on both channels.

\section{PROCEDURE}

The Ss were matched as closely as possible on the basis of MA and IQ and assigned to each of the four experimental groups. The four groups were the following: Group 1-successive presentation, S-R spatially contiguous; Group 2-simultaneous presentation, S-R spatially contiguous; Group 3-successive presentation, S-R spatially separated; and Group 4-simultaneous presentation, S-R spatially separated. The MA and IQ characteristics of all the groups are presented in Table 1.

All Ss were introduced to the experiment as a "candy game" and told that the sounds they heard would tell them where the candy was. Ss in the S-R spatial contiguity groups ( 1 and 2) were told that the sounds would come from the "things (speaker) over the cups." Ss in the S-R spatially separated groups ( 3 and 4 ) were told that the sounds would come from the earphones on their heads and that the things over the cups were there to keep the candy hidden. All correct responses were rewarded with an "M\&M" and a verbal statement of "good" from $E$. Incorrect responses produced a "no" from E. Noncorrection procedure was used throughout.

Table 1 MA and IQ Characteristics of Experimental Groups

\begin{tabular}{ccccr}
\hline & \multicolumn{3}{c}{ MA (in months) } & \multicolumn{2}{c}{ IQ } \\
Group & Mean & SD & Mean & SD \\
\hline 1 & 81.5 & 22.6 & 52.9 & 8.9 \\
2 & 81.5 & 12.5 & 51.7 & 10.4 \\
3 & 82.5 & 21.4 & 55.7 & 9.7 \\
4 & 80.8 & 22.9 & 56.1 & 12.1 \\
\hline
\end{tabular}




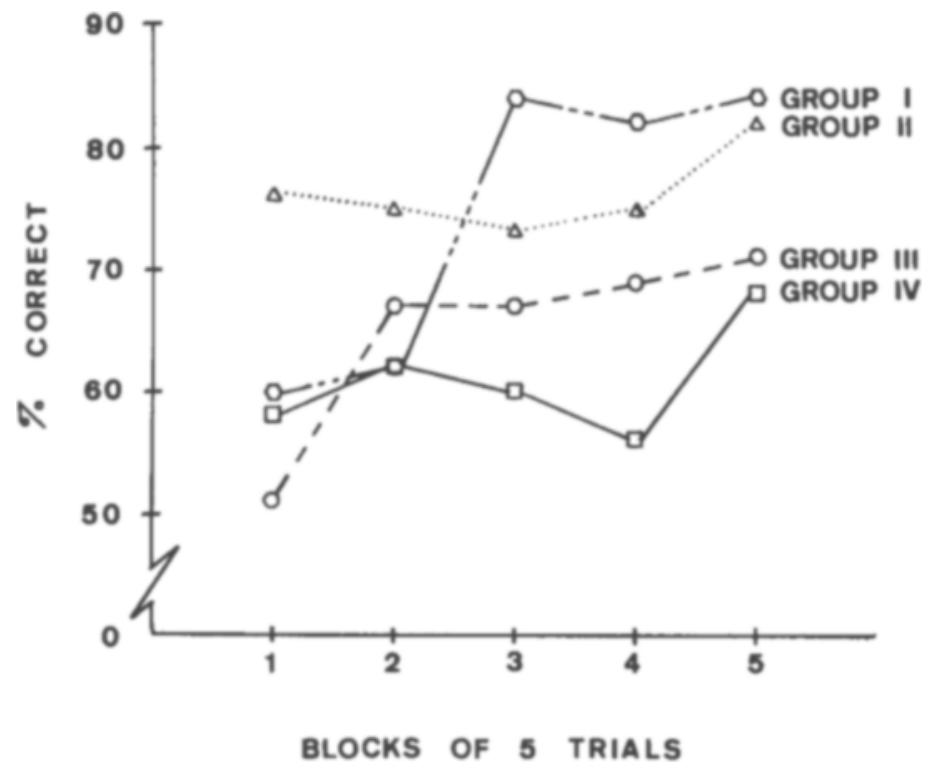

Stimulus onset was produced by E's activation of a switch on the tape deck. A transparent portion of tape marked the offset of the stimulus permitting $E$ to halt the tape during intertrial intervals. Position of the correct stimulus in the simultaneous groups ( 2 and 4 ), and sequence of stimuli in the successive groups ( 1 and 3 ) were determined by a Gellermann series (Gellermann, 1933). Six preprogrammed tapes were prepared: three binaural, for simultaneous conditions and three monaural for successive conditions. The stimulus pairs were whistle-bark, gallop-cymbals, buzzersiren for both binaural and monaural conditions. Each $\mathrm{S}$ was randomly assigned to a particular stimulus pair.

Subjects in all conditions were given a block of 25 trials daily and run to a criterion of 20 of 25 correct. If $S$ failed to reach criterion in 5 days ( 125 trials), no further trials were given.

\section{RESULTS}

Of the $11 \mathrm{Ss}$ in each group, the following number reached criterion: Group 1-7, Group 2-6, Group 3-7, and Group 4-5. Of the $25 \mathrm{Ss}$ reaching criterion, 18 did so in a single block of 25 trials. Therefore, to include the data of all $44 \mathrm{Ss}$, the first day's performance is plotted in blocks of five trials in Fig. 1.

Figure 1 shows that the performance on the first block of five trials is near chance (50\%) for all groups with the exception of Group 2 (simultaneous presentation, S-R spatially contiguous). The functions, overall, reveal an expected increase in performance over blocks of trials with the twoS-R spatially contiguous groups ( 1 and 2$)$ superior to the spatially separated groups (3 and 4). Only small differences exist between successive and simultaneous groups, although the differences under both conditions of spatial contiguity or separation seems to favor the successive presentation.

A 2 by 2 factorial analysis with repeated measures was performed upon the response measure of number correct in a block of five trials.

The analysis reveals that the small successive-simultaneous difference does not reach statistical significance, but that the effect of S-R spatial contiguity as opposed to separateness falls between the .05 and .10 levels $(F=3.81, \mathrm{df}=1 / 40)$. The effect of trials is significantly below the .01 level $(F=3.74, d f=4 / 160)$, and the interaction between trials and simultaneous-successive presentations is significantly below the .05 level $(F=2.48, d f=4 / 160)$. This interactive effect is a function of the high level of the first two trial blocks in Group 2.

\section{DISCUSSION}

While previous research has demonstrated the similarity of retardate visual and auditory discrimination learning (Smith et al, 1967) the present experiment does show a difference in the effects of presentation mode. In the visual situation simultaneous presentation is superior to successive when the stimulus and response are spatially contiguous, with this difference disappearing or reversing when the $S$ and $R$ are spatially separated (Lipsitt, 1961). Figure 1 reveals that in the auditory situation this effect does not seem to occur. Group 2 (simultaneous, S-R contiguity) does not show overall superiority to Group 1 ; indeed, in the final three blocks of trials Group 1 performance is higher. The same successivesimultaneous relation appears to hold in the S-R spatial separation groups, although the main effect of successive superiority over simultaneous is statistically negligible. The absence of simultaneous superiority under
Fig. 1. Per cent correct for four experimental groups.

either condition of spatial S-R relation is congruent with the notion that the auditory training history of the child does not include as high a proportion of simultaneous discriminations as does the visual training history. However, to support the idea that a more extensive training history in successive auditory discrimination is the major determiner of auditory presentation mode effects, a statistically significant successive superiority would be more convincing than the absence of a simultaneous superiority or the trend toward better successive performance observed in the present study.

Just as simultaneous-successive presentation mode effects appear to differ in the auditory and visual situations so does the stimulus-response spatial contiguity effect. The interaction between S-R spatial contiguity and simultaneous-successive presentation found in the visual situation was not found in the present auditory study. Marginal statistical significance was obtained for superiority of S-R spatial contiguity (Groups 1 and 2) over spatially separated S-R (Groups 3 and 4) under both simultaneous and successive conditions. This effect does not fit an intuitive training history explanation as easily as does the lack of simultaneous auditory superiority. It would appear reasonable that children are rewarded for reaching for visually different objects far more often than they are for reaching for different sounds. Such training should diminish the effectiveness of S-R spatial contiguity in the auditory situation, but this was not the case. The effects of auditory S-R spatial contiguity appear worthy of further investigation.

The interaction of the successivesimultaneous variable and trial blocks is puzzling. The trend of performance in successive groups (1 and 3) and simultaneous groups ( 2 and 4 ) appears similar, with the exception of simultaneous Group 2. The first two trial blocks of this group are at a level almost as high as the final trial blocks. No explanation for this effect is immediately apparent. An examination of the MA and $\mathrm{CA}$ characteristics of the experimental groups shows little difference among them, although Group 2 seems to be a bit more homogeneous in MA (SD $=12.5$ ) than do the other three groups. It is unlikely that homogeneity of MA should produce higher initial performance especially in light of the similarity of MA means and IQs.

\section{REFERENCES}

GELLERMAN, L. W. Chance orders of alternating stimuli in visual discrimination experiments. 
Journal of General Psychology, 1933, 42, 207-208.

LIPSITT, L. P. Simultañeous and successive discrimination learning in children. Child Development, 1961, 32, 337-347.

SHEPP, B. Role of distinctive incentives in simultaneous and successive discrimination with retardates. In D. Zeaman et al (Eds.), Learning and transfer in mental defectives. Progress Report No. 2, NIMH USPHS, 1960. Research Grant M-1099 to University of Connecticut, V. 141-151.

SMITH, J., ANDERSON, V., CUNNINGHAM, T., \& SJOBERG, W. A comparison of auditory and visual discrimination learning in retardates.
American Journal of Mental Deficiency, 1967, 72, 445-449.

ZEAMAN, D., \& HOUSE, B. J. The role of attention in retardate discrimination learning. In N. A. Ellis (Ed.), Handbook of mental deficiency, New York: McGraw-Hill, 1961

\section{NOTE}

1. The research reported in this paper was supported - by grants from the University of Connecticut Research Foundation and the U.S. Office of Education. The author is indebted to Drs. David Zeaman and Betty House in whose laboratory the research was conducted.

\section{The effect of post-test relevant pre-tests and discussion-type feedback on learning and retention}

\author{
S. JAY SAMUELS, University of Minnesota, \\ Minneapolis, Minn. 55455
}

The effect of posttest relevant pretest questions and discussion-type feedback on learning and retention was investigated. Ninety-six Ss were randomly assigned to one of three treatments. Experimental Group $A$ got posttest relevant pretest questions and discussion-type feedback. Experimental Group B got the same posttest relevant pretest but not discussion-type feedback. Control Group $C$ got a nonrelevant pretest and no discussion. The test of learning showed Groups $A, B$, and $C$ were all significantly different from each other. On the test of retention Group A maintained its superiority.

A major objective of teaching is to increase student achievement. One way to accomplish this goal is to improve the methodology of instruction. Although tests are usually given for the purpose of measuring student achievement and are infrequently used as an integral part of the instructional method, their use in this latter role may facilitate learning (McKeachie, 1963 , p. 1154). When a test is given as part of the teaching procedure, it may be referred to as a pretest. Pretests may be given with or without feedback and questions on the pretest may or may not be relevant to questions which will be asked on the posttest.
Kellogg \& Bryan (1938) have shown that true-false pretests given without feedback were sufficient to facilitate learning. Similarly, enhanced learning resulted from the use of adjunct questions with a written passage (Hershberger \& Terry, 1965). More recently, Rothkopf (1967) demonstrated that when pretest questions were presented before a passage was read, facilitation was specific to the concepts emphasized by the question; when pretest questions were presented after a passage was read, both question-specific and general facilitative effects were found. Furthermore, Ss who received written answers to questions did better than Ss who received no feedback. Frase (1967) has supported and extended the findings of Rothkopf (1967).

The effect of feedback on learning has been studied by Plowman \& Stroud (1942). They found that students who saw correct copies of their tests eliminated nearly $50 \%$ of their errors upon retest. In the Curtis \& Wood (1929) study, the most beneficial feedback resulted from student correction of their own papers combined with discussion of answers. Sassenrath \& Gaverick (1965) found that discussion-type feedback following an examination was superior to asking students to find answers in their books, and groups getting feedback of any variety were superior to the no-feedback group.

While a number of studies have demonstrated that pretesting and feedback enhance achievement, the effect of discussion-type feedback given in addition to a pretest has not been investigated. The pretest used in this investigation contained page references where students could find answers to the questions.

A second focus of inquiry in this investigation was to determine the effect of asking pretest questions which were directly relevant to questions asked on the posttest. In summary, then, the purpose of this study was to determine the effect of posttest relevant pretest questions and discussiontype feedback on learning and retention.

\section{SUBJECTS}

The Ss were 96 juniors enrolled in Psychological Foundations of Teaching at the University of Minnesota, Summer Session.

\section{DESIGN AND PROCEDURE}

Subjects were randomly assigned to one of three laboratory sections, with 32 to a section. Laboratory instructors and treatments were randomly assigned to sections. All 96 Ss attended the same daily lectures. Following each lecture the students went to their assigned laboratory section. The Ss were not informed that an experiment was taking place. To ensure that the groups were in fact equal, a multiple-choice examination, that was not part of the study, was given. The means for the three groups on the test were: $\quad 30.91 \quad(\mathrm{SD}=2.78), \quad 30.50$ $(\mathrm{SD}=3.23), 31.31(\mathrm{SD}=3.10)$. A one-way analysis of variance on this data showed no significant difference among the groups on this test $(F<1, d f=2 / 93$, n.s. $)$.

For the study, all the Ss were given the same reading assignment from their educational psychology textbook. They were then given in their laboratory sections a 13-item true-false pretest based upon the reading assignment. The cover sheet of the pretest contained information to the student. He was informed that the true-false test was a take-home examination, and that the score he got on the test was to be added to his other examination scores. To help the student in deciding whether his answers were correct, next to each question was a page number indicating where he could look in his textbook for the answer to the question. The students were requested to return test questions and answer sheets to lecture the following day. They were also requested to refrain from discussing their answers with fellow students. Since the pretest was to be graded, it was assumed that the students would take advantage of the opportunity to check their answers.

The treatments for the study were as follows:

EXPERIMENTAL GROUP A: POSTTEST RELEVANT PRETEST WITH DISCUSSION-TYPE FEEDBACK

This group received a 13-item true-false take-home pretest. Next to each true-false 